\title{
Serum miR-21 and miR-92a as biomarkers in the diagnosis and prognosis of colorectal cancer
}

\author{
Guang-Hui Liu, Zong-Guang Zhou, Rong Chen, Mon-Jin Wang, Bin Zhou, Yuan Li and \\ Xiao-Feng Sun
}

\section{Linköping University Post Print}

\section{Tweet}

N.B.: When citing this work, cite the original article.

The original publication is available at www.springerlink.com:

Guang-Hui Liu, Zong-Guang Zhou, Rong Chen, Mon-Jin Wang, Bin Zhou, Yuan Li and Xiao-Feng Sun, Serum miR-21 and miR-92a as biomarkers in the diagnosis and prognosis of colorectal cancer, 2013, Tumor Biology, (34), 4, 2175-2181.

http://dx.doi.org/10.1007/s13277-013-0753-8

Copyright: Karger / Springer Verlag (Germany)

http://www.springerlink.com/?MUD=MP

Postprint available at: Linköping University Electronic Press

http://urn.kb.se/resolve?urn=urn:nbn:se:liu:diva-96414 


\section{Serum miR-21 and miR-92a as biomarkers in the diagnosis and prognosis of colorectal cancer}

Guanghui Liu ${ }^{\mathrm{ab}}$, Zongguang Zhou ${ }^{\mathrm{abc} *}$, Rong Chen ${ }^{\mathrm{ab}}$, Mojin Wang ${ }^{\mathrm{ab}}$, Bin zhou ${ }^{\mathrm{b}}$, Yuan $\mathrm{Li}^{\mathrm{b}}$, Xiaofeng $\operatorname{Sun}^{\mathrm{d}}$

${ }^{a}$ Department of Gastrointestinal Surgery, Sichuan University, Chengdu 610041, China

${ }^{\mathrm{b}}$ Institute of Digestive Surgery, Sichuan University, Chengdu 610041, China

${ }^{\mathrm{c}}$ National Key Laboratory of Biotherapy of West China Hospital, Sichuan University, Chengdu 610041, China

${ }^{\mathrm{d}}$ Division of Oncology, Department of Clinical and Experimental Medicine Faculty of Health Sciences, Country Council of Östergötland, University of Linköping, Linköping S-581 85, Sweden

*Corresponding author: Zongguang Zhou, Department of Gastrointestinal Surgery, West China Hospital, Sichuan University, 37 Guo Xue Xiang, Chengdu 610041, China. Tel: + 86-28-85422004; Fax: + 86-28-85164036; E-mail address: Zhou767@163.com 


\section{Abstract}

Previous studies from our laboratory identified a number of miRNAs that were aberrantly expressed in colorectal cancer (CRC) tissue. However, their diagnostic and prognostic value in serum has not been fully evaluated. In the present study, we measured the levels of 5 miRNAs (miR-21, 31, 92a, 18a and 106a) in serum samples from 200 CRC patients, 50 advanced adenoma patients, and 80 healthy controls by real-time quantitative polymerase chain reaction (RT-PCR). In our study, the levels of miR-21 and miR-92a in patients with CRC and advanced adenoma were significantly higher than those in healthy controls (all $P<$ 0.05). MiR-21 yielded an area under the ROC curve (AUC) of 0.802 and miR-92a yielded an AUC of 0.786 in discriminating CRCs from the controls. Additionally, miR-21 and miR-92a yielded an AUC of 0.709 and 0.701, respectively, in discriminating advanced adenomas from the controls. Combined receiver-operating characteristics (ROC) analyses, using both miRNAs, revealed an elevated AUC of 0.847 in discriminating CRCs, and an AUC of 0.722 in discriminating advanced adenomas from the controls. In the multivariate Cox proportional hazards analysis, high miR-92a expression in CRC was independently associated with poor survival $(P=0.03$; hazard ratio 4.36 ; $95 \%$ confidence interval $=1.64-11.57)$. In summary, our data indicate that miR-21 and miR-92a serum levels have potential value for early detection of CRC. Furthermore, miR-92a has prognostic value in CRC patients. No significant difference was observed in the levels of miR-18a, 31 and 106a among CRC, advanced adenoma and control samples.

Key words: Colorectal cancer; MicroRNA; Serum; Diagnosis; Prognosis 


\section{Abbreviations}

miRNA microRNA

CRC colorectal cancer

AUC area under the ROC curve

ROC receiver-operating characteristics

CEA carcinoembryonic antigen

Ct threshold cycle of PCR amplification 


\section{Introduction}

Colorectal cancer (CRC) is a major cause of cancer-related death worldwide [1], and the incidence and mortality in China have increased rapidly in the past decades [2]. Most CRC-related deaths could be prevented through early diagnosis and surgical removal of early-stage cancer and precancerous lesions. Advanced adenoma, which is an adenoma with significant villous features, a size of $1.0 \mathrm{~cm}$ or more and high-grade dysplasia, bridges benign and malignant states in colorectal tumorigenesis, and represents the optimal target lesion for CRC prevention strategies [3]. Several CRC screening tests, including fecal occult-blood test (FOBT) and colonoscopy, are frequently used in detection of CRC. Nevertheless, none of these tests have been established as a well-accepted screening tool due to their invasiveness, high cost or low sensitivity [4]. Thus, there is an urgent need for new noninvasive biomarkers to improve the detection of CRC. In addition, there is a need to identify new, non-invasive prognostic biomarkers for CRC in order to improve postoperative treatment strategies.

MicroRNAs (miRNAs) are small, non-coding RNAs that regulate the translation of specific protein coding genes. Recent studies have revealed the role of miRNAs in a variety of basic biological and pathological processes[5], and the association of miRNA signatures with human diseases has been established [6, 7]. In 2008, Lawrie et al. [8] first established the existence of miRNAs in circulation. Soon after, circulating miRNAs have been proposed as sensitive and informative biomarkers in the diagnosis and prognosis of several types of cancer [9-12]. Previous studies from our laboratory identified a number of miRNAs that were aberrantly expressed in CRC tissue [13, 14]; however, their diagnostic and prognostic value 
in serum has not been fully evaluated to date.

In this study, we chose 5 miRNAs (miR-21, 31, 92a, 18a and 106a) based on the results obtained from our previous studies [13, 14] and their reported relevance to CRC [15]. Then we examined their expression in serum samples from 200 CRC patients, 50 advanced adenoma patients and 80 healthy volunteers by real-time quantitative polymerase chain reaction (RT-PCR). Furthermore, we investigated the feasibility of using these miRNAs as potential diagnostic and prognostic CRC biomarkers.

\section{Materials and methods}

Study cohort

Following ethical approval and written informed consent, whole blood samples were collected from 200 CRC patients, 50 advanced adenoma patients, and 80 healthy age-matched volunteers, which served as controls. All patients were diagnosed and treated at the West China Hospital in Chengdu, China from November 2006 to June 2008. The patient group is representative of the general distribution of patients from West China Hospital. All blood samples were collected one day before surgery, and all advanced adenomas and CRCs were pathologically confirmed. Patients were excluded if they had any of the following issues: clinical diagnosis of familial adenomatous polyposis or hereditary nonpolyposis CRC, diagnosis of other types of cancer at any site at the time of selection, undergoing chemotherapy or radiotherapy before blood sampling, and receiving blood transfusion in past 
three months. Information regarding the patients' gender, age, tumor location, size, pTNM stage, differentiation, serum carcinoembryonic antigen (CEA), hepatitis B virus (HBV) carrying and cigarette smoking status was obtained from hospital surgical and pathological records. Tumors were staged according to the UICC tumor-node-metastasis (TNM) staging system. The control blood samples were collected from healthy volunteers with no current or previous malignancy, or inflammatory condition. Clinicopathological features of patients are summarized in Supplementary Table 1.

Follow-up

Follow-up of the patients was performed by a combination of outpatient visits, letters, and telephone calls. Patients were asked to perform physical examinations, blood tests, chest radiography and liver ultrasonography every 3-6 months, and colonoscopy annually. Computed tomography or magnetic resonance imaging was performed when required. For the present analysis, the follow-up data were updated in April 2011. The mean follow-up of patients was 36.4 months (range, 4-53 months).

Sample processing and total RNA extraction

Up to $4 \mathrm{ml}$ of whole blood from each participant was collected in a serum separator tube containing EDTA. Samples were left to clot at room temperature for 30 minutes, and then were centrifuged at $4000 \mathrm{rpm}$ for 10 minutes at 4 i̊. $\mathrm{h}$.ermemoved, aliquoted and stored at 
TRIZOL LS (Invitrogen, CA, USA) method according to the manufacturer's protocol. Total RNA concentration and integrity were determined with an ultraviolet specrophotometer (Beckman, CA, USA) and a Digital gel image analysis system (Bio-Rad, CA, USA). The median storage time between blood sample processing for serum and endpoint analysis was 32 months.

Selection of serum miRNA markers

A panel of 5 cancer associated miRNAs (miR-21, 31, 92a, 18a and 106a) was chosen based on the results from our laboratory obtained in previous studies [13, 14] and their reported relevance to CRC [15]. MiR-16 was selected as an internal control [9, 16].

MiRNA quantification by real-time quantitative RT-PCR

Total RNA samples were reverse transcribed (RT) to generate specific cDNAs using primers specific to each miRNA target. Specific cDNAs were then amplified by real-time quantitative RT-PCR using TaqMan miRNA primers and probes purchased from Invitrogen. The sequences of primers and probes are summarized in Supplementary Table 2. The protocol used was designed and validated by the others at our laboratory [13, 14]. Briefly, total RNA from $500 \mu \mathrm{l}$ serum was reverse transcribed with a miRNA RT reaction system and the PCRs were carried out in a final volume of $30 \mu \mathrm{l}$ using an iCycer iQ System (Bio-Rad). All 
reactions, including a no-template control, were run in triplicate. The relative expression of each miRNA was normalized to miR-16 and calculated with the $2^{-\Delta \Delta \mathrm{CT}}$ method [17].

Statistical analysis

MiRNA expression levels were compared using the Mann-Whitney U test or Kruskall-Wallis test. Receiver-operating characteristics (ROC) curves were established to evaluate the diagnostic value of serum miRNAs for differentiating tumors from controls. The overall survival was analyzed by log-rank test, and survival curves were plotted according to Kaplan-Meier. Univariate Cox regression was performed on each clinical covariate to examine its influence on patient survival. Final multivariate models were based on step-wise addition. A Wald statistic of $P<0.05$ was used as the criterion for inclusion in final multivariate models. Data were presented as mean \pm SD. All tests were 2-tailed and results with $P<0.05$ were considered statistically significant. All statistical analyses were performed with SPSS 17.0 software. 


\section{Results}

Expression of miRNAs in serum samples from patients and healthy controls

Expression of the 5 miRNAs was detectable in all analyzed samples. There were no significant differences in serum miRNA levels with respect to gender and age among CRC patients, advanced adenoma patients and healthy controls $(P=0.114, \chi 2$ test; $P=0.934$, ANOVA). MiR-16 expression in the three groups was not significantly different $(P=0.381)$, and was therefore used to normalize the RT-PCR data.

To identify the miRNAs that were up-regulated in serum, we first examined the levels of 5 target miRNAs in CRC, advanced adenoma and control samples. Although the miRNAs were previously identified to be relatively abundant in CRC tissues, our results showed that serum miRNA signatures were not fully consistent with that of solid tumors. No significant difference was observed in the levels of miR-18a, 31 and 106a among CRC, advanced adenoma and control samples $(P=0.093$ for miR-18a, $P=0.190$ for miR-31, and $P=0.214$ for miR-106a). Thus, these three miRNAs were not included in further analyses. The expression of the remaining two miRNAs, miR-21 and miR-92a, was significantly elevated in CRC and advanced adenoma samples when compared with controls (all $P<0.05$, Fig. 1).

Relationship between serum miRNA expression and clinicopathological features

In addition to examining the expression of miRNAs in serum, the relationship between 
miR-92a and miR-21 expression and clinicopathological features of patients was examined. The results showed that high miR-92a expression was associated with advanced pTNM stage $(P<0.001)$ and positive nodal status $(P=0.001)$. In addition, high miR-21 expression was associated with advanced pTNM stage $(\mathrm{P}=0.045)$ and poor differentiation $(P<0.001)$. There were no significant associations between miRNAs and gender, age, tumor location, tumor size, CEA, HBV carrying or cigarette smoking (all $P>0.05$, data not shown).

Expression of miRNAs in serum samples from patients in relation to diagnosis

Since miR-21 and miR-92a levels were significantly elevated in serum of CRC patients in comparison healthy controls, ROC curve analysis was used to explore the potential of using circulating miRNAs as biomarkers for CRC. These analyses revealed that serum levels of both miR-21 and miR-92a were potential biomarkers for differentiating CRC patients from controls with an area under the ROC curve (AUC) of 0.802 [95\% confidence interval (CI) $=$ $0.752-0.852]$ and $0.786(95 \% \mathrm{CI}=0.728-0.845)$, respectively (Fig. 2A and 2B). At a cut-off value of 0.0043 for miR-21, the sensitivity was $65 \%$ and the specificity was $85 \%$. At the cut-off value of 0.00017 for miR-92, the sensitivity was $65.5 \%$ and the specificity was 82.5\%. Combined ROC analyses resulted in an increased AUC of 0.847 (95\% CI $=0.803-$ 0.891 ) with a $68.0 \%$ sensitivity and $91.2 \%$ specificity (Fig. $2 \mathrm{E}$ ).

Since the expression of miRNAs in serum of patients with advanced adenomas was higher than in healthy controls, we further investigated the diagnostic value of serum miR-21 and miR-92a expression levels for detection of early lesions in CRC development. ROC 
curve analyses revealed that both miRNAs might be helpful to differentiate adenomas from controls, with an AUC of 0.709 for miR-21 (95\% CI $=0.618-0.801)$ and 0.901 for miR-92a (95\% CI $=0.610-0.792)$ (Fig. 2C and 2D). Combined ROC analyses resulted in an increased AUC value of 0.722 (95\% CI $=0.633-0.811)$ with a sensitivity of $70.0 \%$ and specificity of $70.0 \%$ (Fig. 2F).

Expression of miRNAs in serum samples from patients in relation to prognosis

Follow-up data was available for 166/200 (83\%) CRC patients included in this study. During the follow-up period 38/166 (23\%) patients died of CRC. Patients with high serum miR-92a expression had a significantly worse prognosis than patients with low expression, with a 3-year overall survival of 52.2\% and 93.8\%, respectively $(P<0.001$, Fig. 3). There was no statistically significant association between miR-21 expression and prognosis $(P=0.126)$.

A Cox proportional hazards analysis was used to further evaluate the potential for serum miR-92a expression as a prognostic biomarker. Univariate survival analyses indicated that miR-92a expression, pTNM stage, differentiation and tumor size were associated with prognosis, while miR-21 expression, gender, age, tumor location, CEA, HBV carrying and cigarette smoking were not. In the multivariate Cox proportional hazards analysis, which included miR-92a, pTNM stage, differentiation and tumor size, high miR-92a expression was independently associated with poor survival $(P=0.03$; $\mathrm{HR}=4.36$; $95 \% \mathrm{CI}=1.64-11.57$; Table 1). 


\section{Discussion}

CRC is one of the leading causes of cancer death worldwide. Although great progress has been made in diagnosis and prognosis in the past decades, there is still a need to improve early detection screening methods and to identify new prognostic biomarkers for CRC [1, 2]. Ideal biomarkers should be easy to measure and have a strong association with clinical outcome. miRNAs are could match these proposed criteria [9-12].

In the present study, miR-21 and miR-92a serum levels in patients with CRC and advanced adenoma were significantly higher than those detected in healthy controls. Both miR-21 and miR-92a were potential biomarkers for CRC and yielded an AUC of 0.802 and 0.786, respectively. Combined ROC analyses revealed an increased AUC of 0.847, indicating the additive effect in the diagnostic value of both miRNAs. Furthermore, both miRNAs were helpful in differentiating advanced adenomas from healthy controls with an AUC of 0.709 for miR-21 and 0.701 for miR-92a. Combined ROC analyses revealed an increased AUC value of 0.722, suggesting their potential value for early detection of CRC. In the multivariate Cox proportional analysis, high miR-92a expression was associated with poor survival in CRC patients independent of tumor staging and differentiation.

MiR-92a is part of the miR-17-92 gene cluster, located at chromosome 13q13. As a known oncomir, the miR-17-92 cluster can promote cell proliferation, suppress apoptosis of cancer cells, induce tumor angiogenesis and accelerate tumor progression [18]. Elevated expression of miR-92a has been observed in CRC [10, 12], lung cancer [19] and thyroid cancer [20], suggesting an important role in tumorigenesis. Ng et al. [12] reported that 
circulating miR-92 was a potential biomarker for CRC diagnosis, which is consistent with our data. Furthermore, our results showed that miR-92a had a prognostic potential, and high miR-92a expression in CRC patients was associated with poor survival. However, contrary results were reported in other diseases. For instance, Tanaka et al. [21] showed that miR-92a was dramatically decreased in the plasma of acute leukemia patients using miR-638 as internal control. Shigoka et al. [22] demonstrated that the amount of miR-92a in plasma from hepatocellular carcinoma patients was decreased compared with that of healthy donors. These inconsistencies may be due to the different diseases and methods used in different studies.

MiR-21 is expressed at high levels in most solid tumors [23]. Studies in human cell lines showed that miR-21 could target tumor suppressor genes, such as PTEN [24] and TPM1 [25]. Our results showed that serum miR-21 had a diagnostic potential for CRC (AUC $=0.802$ ). We found that miR-21 was also a potential diagnostic biomarker (AUC $=0.709$ ) for advanced adenoma. However, although an association of high miR-21 expression in CRC tissue with poor survival was observed by Schetter et al. [6], our results indicated that serum miR-21 expression was not associated with patient survival. These differences may be due to the different samples (serum versus solid tissue) or the different quantification methods used. Ng et al. [12] demonstrated that miR-21 expression in plasma from CRC patients was lower than in healthy controls, which is inconsistent with our current findings. Nevertheless, the results of $\mathrm{Ng}$ et al. have to be taken with caution because their study included only five samples.

MiR-18a and miR-106a also belong to the miR-17-92 cluster [18], while miR-31 is located at chromosome 9p21.3 [26], and these miRNAs are expressed at high levels in CRC 
tissue [12, 14]. CRC patients with high miR-18a expression tended to have a poorer prognosis than the low expression group [15]. High miR-31 expression in CRC tumor tissue was related to advanced pTNM stage [14]. In our study, no significant difference was observed in serum miR-18a, 31 and 106a levels among the CRC patients, advanced adenomas patients and healthy controls. Our results differ from those of other studies, however this difference may be attributed to the different samples (solid tissue versus serum) and methods used.

It has been established that the expression of several miRNAs, including the miR-17-92 cluster [27], miR-31 [28], miR-106a [29], changes in HBV carriers and/or cigarette smokers. China has the largest population of HBV carriers [30] and cigarette smokers [31] in the world. In our study, we examined the possible association of miR-21 and miR-92a expression with HBV status and cigarette smoking in CRC patients; however no association was observed. A possible reason is that the expression of these miRNAs in CRC patients is more influenced by mechanisms involved in tumorigenesis than by HBV infection or cigarette smoking.

One possible concern in this and similar studies of circulating miRNA is the lack of consensus about internal controls for quantitative RT-PCR. Several internal controls have been used thus far in different tumor types, such as miR-16 [9], miR-197 [32], 5S RNA [14] and RNU6B [12]. The results of this study show that miR-16 expression in serum is similar across the patient and control groups, and therefore can be used as a reliable internal control in CRC miRNA expression studies. Secondly, all patients were from the southwestern part of China and most of the patients were relatively poor with high turnover due to work that led to the lack of standard postoperative therapy, regular examination and high-rate follow-up. 
Therefore, we were not able to collect enough data to analyze disease-free survival and therapy response in relation to the miRNAs.

In conclusion, miR-21 and miR-92a have potential as non-invasive biomarkers for early detection of CRC. Furthermore, high miR-92a expression is independently associated with poor survival in CRC patients and may be used in the future as a potential prognostic biomarker. 


\section{Acknowledgments}

We thank the colleagues of the Department of Gastrointestinal Surgery and Institute of Digestive Surgery for providing blood samples and surgical/pathological records. This study was supported by the National Natural Science Foundation of China (No.30830103).

\section{Role of the funding source}

The National Natural Science Foundation of China was not involved in the study design, collection, analysis and interpretation of data, writing of the report, and decision making to submit the paper for publication. 


\section{References}

1. Jemal A, Siegel R, Ward E, Hao Y, Xu J, Thun MJ. Cancer statistics, 2009. CA Cancer. J Clin. 2009;59:225-49.

2. Yang L, Parkin DM, Li L, Chen Y. Time trends in cancer mortality in China: 1987-1999.Int J Cancer. 2003;106:771-83.

3. S.J. Winawer, A.G. Zauber.The advanced adenoma as the primary target of screening. Gastrointest Endosc Clin N Am. 2002;12:1-9.

4. Levin B, Lieberman DA, McFarland B, Smith RA, Brooks D, Andrews KS, et al. Screening and surveillance for the early detection of colorectal cancer and adenomatous polyps, 2008: a joint guideline from the American Cancer Society, the US Multi-Society Task Force on Colorectal Cancer, and the American College of Radiology. CA Cancer J Clin. 2008;58:130-60.

5. Lu J, Getz G, Miska EA, Alvarez-Saavedra E, Lamb J, Peck D, et al. MicroRNA expression profiles classify human cancers. Nature. 2005;435:834-8.

6. Schetter AJ, Leung SY, Sohn JJ, Zanetti KA, Bowman ED, Yanaihara N, et al. MicroRNA expression profiles associated with prognosis and therapeutic outcome in colon adenocarcinoma. JAMA. 2008;299:425-36.

7. Baraniskin A, Kuhnhenn J, Schlegel U, Chan A, Deckert M, Gold R, et al. Identification of microRNAs in the cerebrospinal fluid as marker for primary diffuse large B-cell lymphoma of the central nervous system. Blood. 2011;117:3140-6.

8. Lawrie CH, Gal S, Dunlop HM, Pushkaran B, Liggins AP, Pulford K, et al. Detection of elevated levels of tumor-associated microRNAs in serum of patients with diffuse large B-cell lymphoma. Br J Haematol. 2008;141:672-5.

9. Heneghan HM, Miller N, Lowery AJ, Sweeney KJ, Newell J, Kerin MJ. Circulating microRNAs as novel minimally invasive biomarkers for breast cancer. Ann Surg. 
2010;251:499-505.

10. Miyachi M, Tsuchiya K, Yoshida H, Yagyu S, Kikuchi K, Misawa A, et al. Circulating muscle-specific microRNA, miR-206, as a potential diagnostic marker for rhabdomyosarcoma. Biochem Biophys Res Commun. 2010;400:89-93.

11. Li J, Wang Y, Yu W, Chen J, Luo J. Expression of serum miR-221 in human hepatocellular carcinoma and its prognostic significance. Biochem Biophys Res Commun. 2011;406:70-3.

12. Ng EK, Chong WW, Jin H, Lam EK, Shin VY, Yu J, et al. Differential expression of microRNAs in plasma of colorectal cancer patients: a potential marker for colorectal cancer screening. Gut. 2009;58:1375-81.

13. Ma Q, Yang L, Wang C, Yu YY, Zhou B, Zhou ZG. Differential expression of colon cancer microRNA in microarray study. J Sichuan Med Sci Ed. 2011;42:344-8.

14. Wang CJ, Zhou ZG, Wang L, Yang L, Zhou B, Gu J, et al. Clinicopathological significance of microRNA-31, -143 and -145 expression in colorectal cancer. Dis Markers. 2009;26:27-34.

15. Motoyama K, Inoue H, Takatsuno Y, Tanaka F, Mimori K, Uetake H, et al. Over- and under-expressed microRNAs in human colorectal cancer. Int J Oncol. 2009;34:1069-75.

16. Mitchell PS, Parkin RK, Kroh EM, Fritz BR, Wyman SK, Pogosova-Agadjanyan EL, et al. Circulating microRNAs as stable blood-based markers for cancer detection. Proc Natl Acad Sci U S A. 2008;105:10513-8.

17. Schmittgen TD, Livak KJ. Analyzing real-time PCR data by the comparative CT method- Nat. Protoc. 2008;3:110-8.

18. Mendell JT. MiRiad roles for the miR-17-92 cluster in development and disease. Cell. 2008;133:217-22.

19. Matsubara H, Takeuchi T, Nishikawa E, Yanagisawa K, Hayashita Y, Ebi H, et al. 
Apoptosis induction by antisense oligonucleotides against miR-17-5p and miR-20a in lung cancers overexpressing miR-17-92. Oncogene. 2007;26:6099-105.

20. Taguchi A, Yanagisawa K, Tanaka M, Cao K, Matsuyama Y, Goto H, et al. Identification of hypoxia-inducible factor-1 alpha as a novel target for miR-17-92 microRNA cluster. Cancer Res. 2008;68:5540-5.

21. Tanaka M, Oikawa K, Takanashi M, Kudo M, Ohyashiki J, Ohyashiki K, et al. Down-regulation of miR-92 in human plasma is a novel marker for acute leukemia patients. PLoS One. 2009;4:e5532.

22. Shigoka M, Tsuchida A, Matsudo T, Nagakawa Y, Saito H, Suzuki Y, et al. Deregulation of miR-92a expression is implicated in hepatocellular carcinoma development. Pathol Int. 2010;60:351-7.

23. Volinia S, Calin GA, Liu CG, Ambs S, Cimmino A, Petrocca F, et al. A microRNA expression signature of human solid tumors defines cancer gene targets. Proc Natl Acad Sci U S A. 2006;103:2257-61.

24. Meng F, Henson R, Lang M, Wehbe H, Maheshwari S, Mendell JT, et al. Involvement of human micro-RNA in growth and response to chemotherapy in human cholangiocarcinoma cell lines. Gastroenterology. 2006;130:2113-29.

25. Zhu S, Si ML, Wu H, Mo YY. MicroRNA-21 targets the tumor suppressor gene tropomyosin 1 (TPM1).J Biol Chem. 2007;282:14328-36.

26. Lagos-Quintana M, Rauhut R, Lendeckel W, Tuschl T. Identification of novel genes coding for small expressed RNAs. Science. 2001;294:853-8.

27. Liu WH, Yeh SH, Chen PJ. Role of microRNAs in hepatitis B virus replication and pathogenesis. Biochim Biophys Acta. 2011;1809:678-85.

28. Izzotti A, Larghero P, Cartiglia C, Longobardi M, Pfeffer U, Steele VE, et al. Modulation of microRNA expression by budesonide, phenethyl isothiocyanate and cigarette smoke in 
mouse liver and lung. Carcinogenesis. 2010;31:894-901.

29. Ji F, Yang B, Peng X. Circulating microRNAs in hepatitis B virus-infected patients. J Viral Hepatitis. 2011;18:e242-e51.

30. Zhu CT. Dong CL. Characteristics of general distribution of hepatitis B virus genotypes in China. Hepatobiliary Pancreat Dis Int. 2009;8:397-401.

31. Lin Y, Fraser T. A review of smoke-free health care in mainland China. Int J Tuberc Lung Dis. 2011;15:453-8.

32. Ruebel K, Leontovich AA, Stilling GA, Zhang S, Righi A, Jin L, et al. MicroRNA expression in ileal carcinoid tumors downregulation of microRNA-133a with tumor progression Modern. Pathol. 2010;23:367-75. 


\section{Figures Legends}
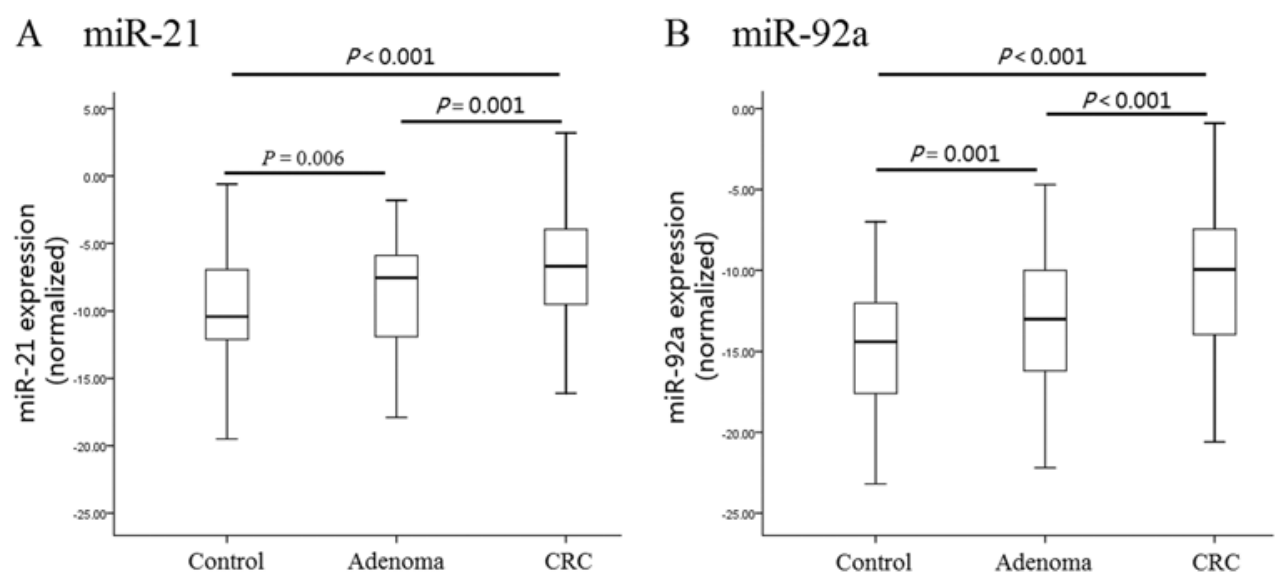

Fig 1. Serum levels of miR-21 (A) and miR-92a (B) from CRC patients, advanced adenoma patients and healthy controls. The lines inside the boxes denote the medians. Expression levels of the miRNAs are normalized to miR-16. 

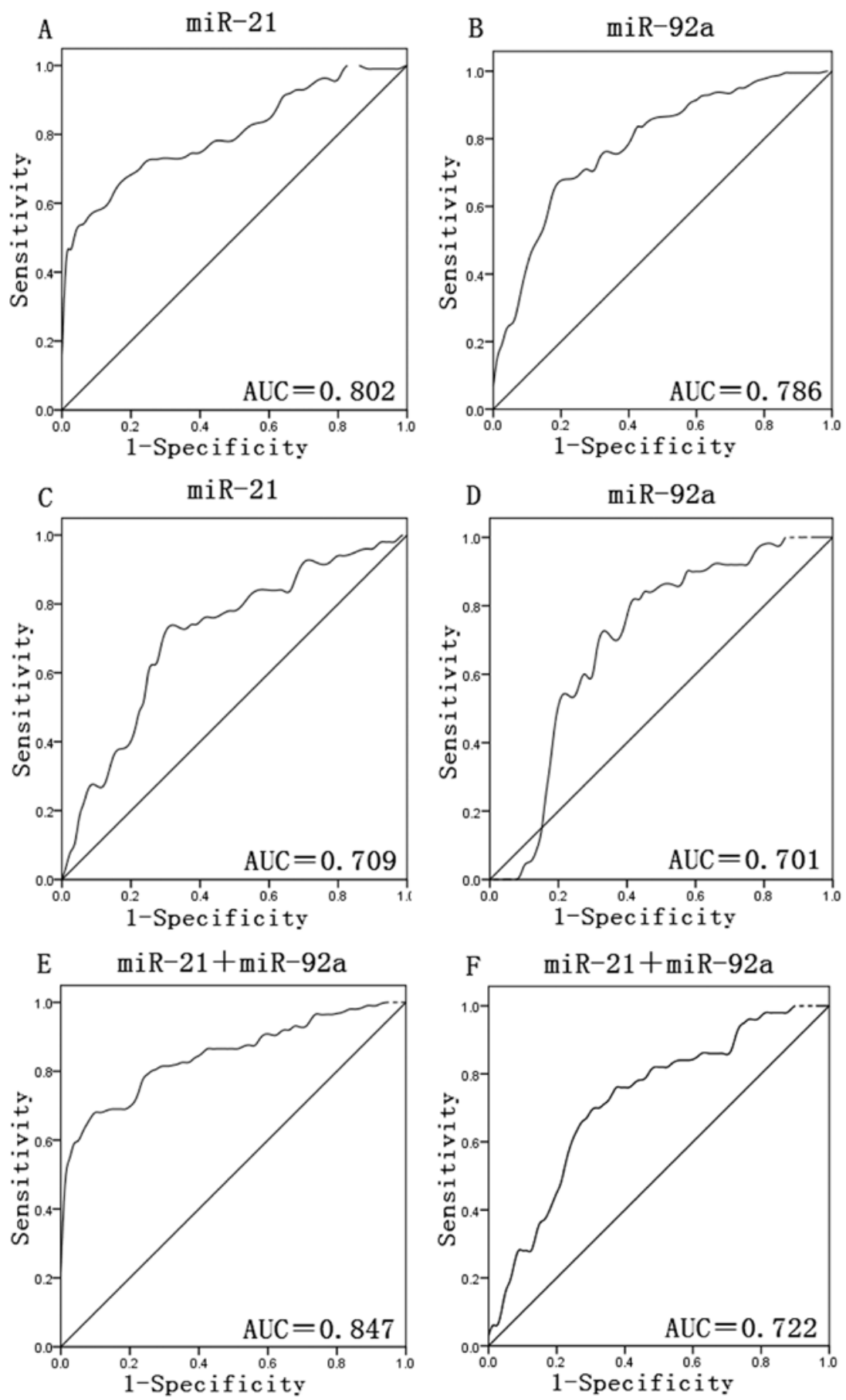

Fig 2. Receiver operating characteristics (ROC) curve analysis using serum miR-21 and miR-92a for discriminating CRCs from healthy controls. (A) ROC curve for miR-21 in discriminating CRCs. (B) ROC curve for miR-92a in discriminating CRCs. (C) ROC curve for miR-21 in discriminating adenomas. (D) ROC curve for miR-92a in discriminating adenomas. (E) Combined ROC curve for miR-21 and miR-92a in discriminating CRCs. (F) Combined ROC curve for miR-21 and miR-92a in discriminating adenomas. 


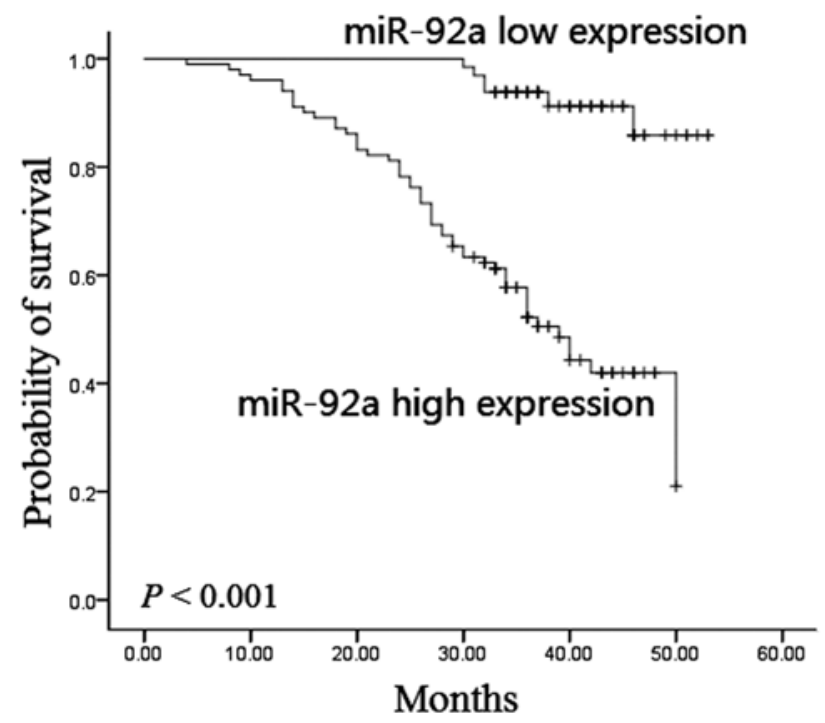

Fig 3. MiR-92a expression in serum in relation to the survival of CRC patients $(P<0.001$, Log-rank test, Kaplan Meier curve). 
Table 1. Univariate and multivariate Cox regression analysis of miR-92a expression levels and prognosis of patients with CRC

\begin{tabular}{|c|c|c|c|c|c|}
\hline & \multirow{2}{*}{ Characteristics } & \multicolumn{2}{|c|}{ Univariate Analysis } & \multicolumn{2}{|c|}{ Multivariate Analysis } \\
\hline & & HR $(95 \%$ CI) & $P$ value $^{\mathrm{a}}$ & HR $(95 \%$ CI) & $P$ value $^{\mathrm{b}}$ \\
\hline MiR-92a & Down/Up & $\begin{array}{c}10.19(4.05-25 . \\
65)\end{array}$ & $<0.001$ & 4.36(1.64-11.57) & 0.03 \\
\hline TNM stage & I /II/II/IV & $3.12(2.23-4.36)$ & $<0.001$ & $2.29(1.58-3.31)$ & $<0.001$ \\
\hline Differentiation & Well/Moderately/Poorly/Undifferentiated ${ }^{\mathrm{c}}$ & $3.39(2.47-4.64)$ & $<0.001$ & $2.65(1.90-3.70)$ & $<0.001$ \\
\hline MiR-21 & Down/Up & $1.58(0.77-3.21)$ & 0.126 & & \\
\hline Gender & Male/Female & $0.96(0.57-1.64)$ & 0.897 & & \\
\hline Age (year) & $\leq 50 />50$ & $1.04(0.64-1.79)$ & 0.947 & & \\
\hline Cigarette smoking & Negative/Positive & $0.97(0.53-1.77)$ & 0.684 & & \\
\hline Tumor location & Right/Left/Rectum & $0.94(0.68-1.30)$ & 0.935 & & \\
\hline Tumor size (cm) & $\leq 5 />5$ & $2.44(1.45-4.12)$ & $<0.001$ & & \\
\hline CEA (ng/ml) & $\leq 3.4 />3.4$ & $1.38(0.82-2.33)$ & 0.2 & & \\
\hline HBV & Negative/Positive & $1.39(0.77-2.51)$ & 0.243 & & \\
\hline
\end{tabular}

Abbreviation: CRC, colorectal cancer; HR, hazard ratio, CEA, carcinoembryonic antigen; HBV, hepatitis B virus.

${ }^{\mathrm{a}}$ Kaplan-Meier method. ${ }^{\mathrm{b}}$ Multivariate Cox proportional hazard.

${ }^{\mathrm{c}}$ Signet ring cell and mucinous adenocarcinoma were included. 
Supplementary Table 1. Summary of clinicopathological features of patients used for miRNA analysis

\begin{tabular}{|c|c|c|c|c|}
\hline \multicolumn{2}{|c|}{ Characteristics } & \multirow{2}{*}{$\begin{array}{c}\text { CRCs }(n=200) \\
126 / 74\end{array}$} & \multirow{2}{*}{$\begin{array}{c}\text { Advanced adenomas }(\mathrm{n}=50) \\
25 / 25\end{array}$} & \multirow{2}{*}{$\begin{array}{c}\text { Healthy controls }(\mathrm{n}=80) \\
42 / 38\end{array}$} \\
\hline Gender & Male/Female & & & \\
\hline Age & Mean (Range) (years) & $57.09(20,89)$ & $57.38(38,77)$ & $57.71(28,89)$ \\
\hline Cigarette smoking & Yes/No & $161 / 39$ & & \\
\hline Tumor location & Right/ Left/ Rectum & $43 / 37 / 120$ & & \\
\hline Tumor size & $\leq 5 \mathrm{~cm} />5 \mathrm{~cm}$ & $147 / 53$ & & \\
\hline pTNM stage & I/II/III/IV & $18 / 96 / 64 / 22$ & & \\
\hline Differentiation & $\begin{array}{l}\text { Well/Moderately/ Poorly } \\
\text { /undifferentiated }\end{array}$ & $7 / 134 / 3 / 24$ & & \\
\hline CEA & $\leq 3.4 />3.4(\mathrm{ng} / \mathrm{ml})$ & $103 / 97$ & & \\
\hline HBV carrying & Negative/Positive & $140 / 60$ & & \\
\hline
\end{tabular}

Abbreviations: CRC, colorectal cancer; CEA, carcinoembryonic antigen; HBV, hepatitis B virus.

${ }^{a}$ Signet ring cell and mucinous adenocarcinoma were included. 
Supplementary Table 2. Sequences of primers and probes for miRNAs

\begin{tabular}{ccc}
\hline \multicolumn{2}{c}{ MiRNA } & Sequence \\
\hline MiR-16 & RT primer & 5'-GTCGTATCCAGTGCTGGGTCCGAGTGATTCGCACTGGATACGACCGCCAATA-3' \\
& Forward primer & 5'-GCGTAGCAGCACGTAAATAT-3' \\
MiR-18a & RT primer & 5'-GTCGTATCCAGTGCTGGGTCCGAGTGATTCGCACTGGATACGCTATCTG-3' \\
& Forward primer & 5'-AAGGTGCATCTAGTGCAGATA-3' \\
MiR-21 & RT primer & 5'-GTCGTATCCAGTGCTGGGTCCGAGTGATTCGCACTGGATACGACTCAACATC-3' \\
& Forward primer & 5'-GCTTCGCCTAGCTTATACAGACT-3' \\
MiR-31 & RT primer & 5'-GTCGTATCCAGTGCTGGGTCCGAGTGATTCGCACTGGATACGACCAGCTA-3' \\
& Forward primer & 5'-ACGCGGCAAGATGCTGGCA-3' \\
MiR-92a & RT primer & 5'-GTCGTATCCAGTGCTGGGTCCGAGTGATTCGCGCTGGATACGACACAGGCCG-3' \\
& Forward primer & 5'-CCCTGTATTGCACTTGTCC-3' \\
MiR-106a & RT primer & 5'-GTCGTATCCAGTGCTGGGTCCGAGTGATTCGCACTGGATACGCTACCTG-3' \\
& Forward primer & 5'-GCGAAAAGTGCTTACAGTGC-3' \\
Reverse primer (universal) & 5'-CAGTGCTGGGTCCGAGTGA-3' \\
TaqMan probe (universal) & 5'-FAM-CCCGACCCTGCTTAGCTTCCGA-TAMRA-3' \\
\hline
\end{tabular}

